\title{
Emissive Langmuir Probes in the Strong Emission Regime for the Determination of the Plasma Properties
}

\author{
S. P. Tierno, J. L. Domenech-Garret, J. M. Donoso, D. Jennewein, G. Herdrich, S. Fasoulas, and L. Conde
}

\begin{abstract}
The determination of the plasma potential $V_{\mathrm{pl}}$ of unmagnetized plasmas by using the floating potential of emissive Langmuir probes operated in the strong emission regime is investigated. The experiments evidence that, for most cases, the electron thermionic emission is orders of magnitude larger than the plasma thermal electron current. The temperature-dependent floating potentials of negatively biased $V_{p}<V_{\mathrm{pl}}$ emissive probes are in agreement with the predictions of a simple phenomenological model that considers, in addition to the plasma electrons, an additional electron group that contributes to the probe current. The latter would be constituted by a fraction of the repelled electron thermionic current, which might return back to the probe with a different energy spectrum. Its origin would be a plasma potential well formed in the plasma sheath around the probe, acting as a virtual cathode or by collisions and electron thermalization processes. These results suggest that, for probe bias voltages close to the plasma potential $V_{p} \sim V_{\mathrm{pl}}$, two electron populations coexist, i.e., the electrons from the plasma with temperature $T_{e}$ and a large group of returned thermionic electrons. These results question the theoretical possibility of measuring the electron temperature by using emissive probes biased to potentials $V_{p} \lesssim V_{\mathrm{pl}}$.
\end{abstract}

Index Terms-Electron emission, plasma diagnostics, plasma probes, plasma properties.

\section{INTRODUCTION}

$\mathbf{T}$ HE collecting and emissive Langmuir probes to measure the local properties of cold low-density plasmas are widespread experimental techniques [1], [2]. The collecting probes are passive metallic electrodes with well-defined shapes immersed in the plasma that, according to its bias potential $V_{p}$, collects an electric current $I_{p}\left(V_{p}\right)$ composed of electrons and ions. When the electron energy distribution function could be approximated by a local Maxwellian, plasma potential $V_{\mathrm{pl}}$, electron density $n_{e}$, and temperature $T_{e}$ could be determined from these current-voltage ( $I-V)$ characteristic curves [1], [2].
However, in a number of situations of interest, the electron energy spectra are not Maxwellian, as for drifting plasmas or when different electron populations are present. In this last case, each electron group contributes to the probe current and complicates the interpretation of the measurements using collecting probes.

Nevertheless, the collecting Langmuir probes are still useful when they are operated in conjunction with emissive probes, which are less sensitive to the electron energy spectrum [2]-[4]. The emissive probes are currently made up of a thin tungsten wire exposed to the plasma and heated by a dc or an ac $I_{H}$ up to temperatures over $T_{p}>2000 \mathrm{~K}$. Therefore, the low passive collected current superposes to the temperaturedependent thermionic electron current density $j_{\mathrm{rd}}\left(T_{p}\right)$ given by the Richardson-Dushman expression [2]-[4]

$$
j_{\mathrm{rd}}\left(T_{p}\right)=A T_{p}^{2} \exp \left(-\frac{e W_{f}}{k_{B} T_{p}}\right)
$$

where $W_{f}$ is the metal work function, and $A=6.02 \times$ $10^{5} \mathrm{~A} / \mathrm{m}^{2} \mathrm{~K}^{2}$ is a constant [1].

The electrons are emitted and repelled by the probe for bias potentials $V_{p}<V_{\mathrm{pl}}$, whereas they are attracted for $V_{p}>V_{\mathrm{pl}}$. Hence, the value of the plasma potential could be determined by the steep transition observed in the probe current $I_{p}\left(T_{p}, V_{p}\right)$ between the electron collection and emission processes, which is enhanced by the probe thermionic current. Therefore, the emissive probe introduces an additional electron group that inevitably perturbs the local plasma. In accordance to probe temperature $T_{p}$, which is roughly controlled by heating current $I_{H}$, the emissive probes operate in the weak or strong electron emission regimes. The ratio

$$
R=j_{\mathrm{rd}}\left(T_{p}\right) / j_{\mathrm{eo}}\left(T_{e}, n_{e}\right)
$$

characterizes the magnitude of the emitted thermionic current $j_{\text {rd }}\left(T_{p}\right)$ compared with the electron thermal current density $j_{\mathrm{eo}}\left(T_{e}, n_{e}\right)=e n_{e} V_{\mathrm{eT}} / 4$, with $V_{\mathrm{eT}}$ being the electron thermal speed.

The weak emission regime, where $R \ll 1$, corresponds to moderate probe temperatures (or equivalently, low $I_{H}$ ). In this case, the so-called inflection point method determines the plasma potential by using the maximum of the derivative $d I_{p}\left(T_{p}, V_{p}\right) / d V_{p}$ of the current collected in the limit of low $T_{p}$ [2]-[5]. However, this derivation process becomes often difficult because of the poor signal-to-noise ratio in $I_{p}\left(T_{p}, V_{p}\right)$ 
caused by the combination of the small wire surface and the low thermionic current.

When the emissive probe operates in the strong emission regime (high currents $I_{H}$ ), the electron thermionic current increases, and hence, the $I_{p}\left(T_{p}, V_{p}\right)$ current grows. In these conditions, the floating potential $I_{p}\left(T_{p}, V_{F}\right)=0$ of the probe in a Maxwellian plasma for $V_{F}\left(T_{p}\right) \lesssim V_{\mathrm{pl}}$ is given by [8]-[10]

$$
V_{F}\left(T_{p}\right)-V_{\mathrm{pl}}=\frac{k_{B} T_{e}}{e} \ln \left[\frac{j_{\mathrm{ai}}\left(V_{F}, T_{i}\right)+j_{\mathrm{rd}}\left(T_{p}\right)}{j_{\mathrm{eo}}\left(T_{e}, n_{e}\right)}\right]
$$

where $j_{\mathrm{ai}}\left(V_{F}, T_{i}\right) \ll j_{\mathrm{eo}}\left(T_{e}, n_{e}\right)$ is the small current density of attracted ions having a Maxwellian distribution with the temperature $T_{i}$. Equation (3) is the theoretical basis of the floatingpoint method. Ratio $R$ depends on the probe temperature, and when $T_{p}$ becomes high enough, $R \sim 1$ and the floating potential $V_{F}\left(T_{p}\right) \simeq V_{\mathrm{pl}}$ in (3). This technique is popular because the measurement of the floating potential of a hot probe is much simpler than to derive the full $I-V$ curve.

In this paper, we analyze the magnitude of the perturbation introduced in the plasma by emissive probes operated in the strong emission regime. The estimation of the probe temperature $T_{p}$ instead of using $I_{H}$ allows us to determine the magnitude of the thermionic current $j_{\mathrm{rd}}\left(T_{p}\right)$ and therefore the precise determination of the different probe operation modes.

As we shall see, the experimental evidence points out that (3) is not always satisfied because the probe temperatures $T_{\mathrm{pl}}$, where $V_{F}\left(T_{\mathrm{pl}}\right) \simeq V_{\mathrm{pl}}$, give $R \gtrsim 10^{2}-10^{3}$ in (2). The limits for such strong emission regime are not accurately determined although it is currently accepted that $R \gg 1$ when the spacecharge effects become appreciable [2], [6]-[8]. The probe floats at the plasma potential for emitted thermionic electron currents orders of magnitude higher than those corresponding to $R \sim 1$. Therefore, this lies outside the range of $R \lesssim 1$ and $V_{F}-$ $V_{\mathrm{pl}} \leq 0$, where (3) remains valid.

Nevertheless, the floating potential of hot emissive probes with $T_{p} \simeq T_{\mathrm{pl}}$, where $R \gg 1$, provides reliable measurements of the plasma potential despite the large local perturbation introduced in the plasma. We found that the measurements of $V_{F}\left(T_{p}\right)$ are in good agreement with the calculations of a simple phenomenological model that accounts for the large thermionic currents. Equation (3) is recovered from our model in the appropriate limit, and this would explain the discrepancies found between (3) and the operation regime of the floating emissive probe for $V_{F}-V_{\mathrm{pl}} \leq 0$.

\section{EXPERIMENTAL SETUP}

The plasmas were produced by means of a ring cusp ion plasma thruster described in [12], which was operated as a plasma source. This device produces plasmas with electron densities of $n_{e} \sim 0.1-8 \times 10^{8} \mathrm{~cm}^{-3}$ and electron temperatures between $T_{e} \sim 1-2 \mathrm{eV}$. The neutral gas pressures inside the vacuum chamber were in the range of $8 \times 10^{-4}$ and $3 \times$ $10^{-3} \mathrm{mBar}$ of argon that were held constant by means of a Bronkhorst F-210C leak valve driven by the E7100 controller.

Two Langmuir probes, one collecting and another emissive, were placed at a fixed point distant $24 \mathrm{~cm}$ away from the plasma

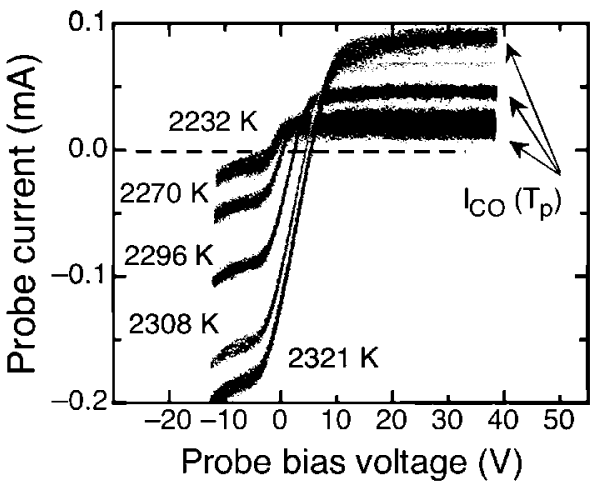

Fig. 1. Current-voltage curves $I_{p}\left(V_{p}, T_{p}\right)$ of an emissive Langmuir probe for increasing wire temperatures $T_{p}$. The arrows indicate the saturation current $I_{\mathrm{co}}\left(T_{p}\right)$ for probe bias voltages $V_{p} \gg V_{\mathrm{pl}}$, and the dashed line corresponds to $I_{p}\left(V_{F}, T_{p}\right)=0$.

source. The collecting probe was made of a stainless-steel ball of $4.38 \mathrm{~mm}$ in diameter, and the emissive probe was a small loop of a thin tungsten wire (with $d=0.08 \mathrm{~mm}$ in diameter, $L=11.4 \mathrm{~mm}$ of length, and surface $S=\pi d L$ ) located at the end of an insulated ceramic tube. The collecting and emissive probes were separated by $10 \mathrm{~mm}$, whereas the involved Debye lengths were $\lambda_{D} \sim 0.3-2.4 \mathrm{~mm}$. Therefore, the collecting and emissive probes were as close as possible to allow the cross check of their respective measurements but distant enough to avoid mutual interference.

The temperature $T_{p}$ of the emissive probe determines the thermionic electron emission through its surface. This plasma exposed wire was heated up to temperatures within the range of $1990 \mathrm{~K}-2440 \mathrm{~K}$ by dc's between $I_{H}=0.75$ and $1.10 \mathrm{~A}$ $\mathrm{dc}$ using a stabilized power supply. The typical operation temperatures are over $2000 \mathrm{~K}$ and could be estimated by means of a classical approximated expression. For a tungsten wire of diameter $d$ in centimeters heated by a dc $I_{H}$ in amperes

$$
T_{p}=T_{0}+P_{1}\left(\frac{I_{H}}{d^{3 / 2}}\right)^{P_{2}} \text {. }
$$

This empirical expression, where $T_{0}=204.35 \mathrm{~K}, P_{1}=$ $29.50 \mathrm{~K}$, and $P_{2}=0.59$ are parameters obtained from the experimental data fitting, provides values for the wire temperature within $\pm 15 \mathrm{~K}[11]$.

The typical voltage current curves of an emissive probe in the strong emission regime for different probe temperatures $T_{p}$ are shown in Fig. 1. These $I-V$ characteristic curves for both collecting $I_{p}\left(V_{p}\right)$ and emissive $I_{p}\left(T_{p}, V_{p}\right)$ probes were obtained by means of a fast sweep biasing circuit discussed in [13]. Briefly, the probes are biased with voltage ramp signals with a repetition pulse over $2.0 \mathrm{kHz}$, and the values of the probe current and bias voltage were later digitized by means of a Yokogawa DL9140 digital oscilloscope for the subsequent analysis.

The circuit in Fig. 2 was employed for the direct measurement of the floating potential $V_{F}\left(T_{p}\right)$ of the emissive probe as a function its temperature. The probe is heated up by a dc $I_{H}$, and because the voltage drops between the resistors $R$ are equal, point $M$ has the same voltage with the midpoint of the emissive probe wire. The voltage $V_{\mathrm{ps}}$ of the precision power supply is 


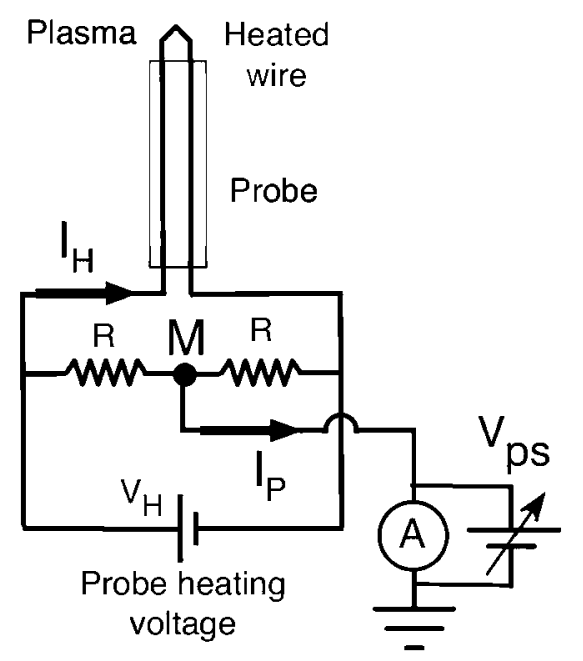

Fig. 2. Circuit for the measurement of the floating potential of the emissive probe heated by the dc $I_{H}$. The value of the resistances is $R=14.5 \mathrm{M} \Omega, A$ is an ammeter, and $V_{\mathrm{ps}}$ is the voltage of a precision power supply.

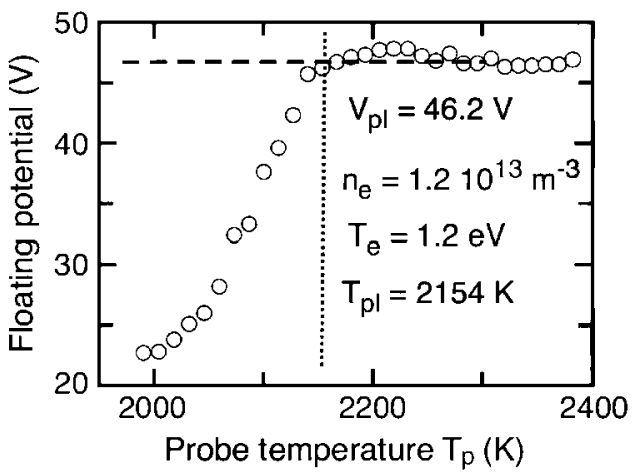

Fig. 3. Measurements of the floating potential $V_{F}\left(T_{p}\right)$ for different wire temperatures $T_{p}$. The dashed line indicates that the plasma potential $V_{\mathrm{pl}} \simeq$ $V_{F}\left(T_{\mathrm{pl}}\right)$ is reached at the transition temperature $T_{\mathrm{pl}}$ marked by the vertical dotted line.

adjusted up to the value of the floating potential $V_{\mathrm{ps}}=V_{F}\left(T_{p}\right)$, where the current $I_{p}\left(T_{p}, V_{F}\right)=0$ between $M$ and the ground point becomes null.

\section{EXPERIMENTAL RESULTS}

In the experiments, electron densities $n_{e}$ and temperatures $T_{e}$ were measured using the collecting probe and the plasma potential. Later, the values of $V_{\mathrm{pl}}$ were always cross checked against the measurements of the emissive probe. The typical floating potentials $V_{F}\left(T_{p}\right)$ as a function of the probe temperature $T_{p}$ are represented in Fig. 3. The values of $V_{F}\left(T_{p}\right)$ approach the plasma potential $V_{\mathrm{pl}}$ as the thermionic electron emission increments. Probe temperature $T_{\mathrm{pl}}$, where $V_{F}\left(T_{\mathrm{pl}}\right) \simeq$ $V_{\mathrm{pl}}$, corresponds to the abrupt change in the slope observed in the experimental data. Further increments in $T_{p}>T_{\mathrm{pl}}$ smoothly increase $V_{F}\left(T_{p}\right)>V_{\mathrm{pl}}$ by a few volts, as shown in Fig. 3 .

In Fig. 4, the calculated ratios $R$ of (2) for the probe temperatures in Fig. 3 are represented, which evidence that $R \sim 10^{2}-10^{3}$ when $T_{p}$ becomes close to $T_{\mathrm{pl}}$. Therefore, when $V_{F}\left(T_{\mathrm{pl}}\right) \simeq V_{\mathrm{pl}}$, the thermionic electron current densities $j_{\mathrm{rd}}\left(T_{p}\right)$ are always between two and three orders of magnitude

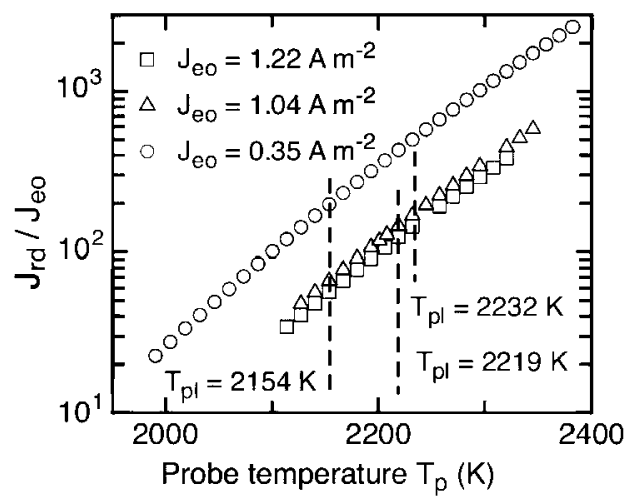

Fig. 4. Ratios $R$ between thermionic electron current $j_{\mathrm{rd}}\left(T_{p}\right)$ and plasma electron thermal current $j_{\mathrm{eo}}\left(T_{e}, n_{e}\right)$. The dashed lines indicate transition temperature $T_{\mathrm{pl}}$, where $V_{F}\left(T_{\mathrm{pl}}\right) \simeq V_{\mathrm{pl}}$, as shown in Fig. 2 for different experiments.

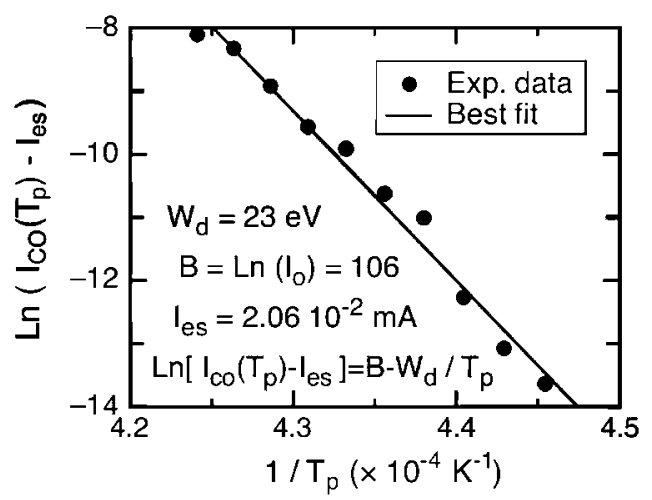

Fig. 5. Best fit of $I_{\mathrm{CO}}\left(T_{p}\right)-I_{\mathrm{eS}}$ to (5), where $I_{\mathrm{ES}}$ is the saturation current for the lower $T_{p}$ of the $I-V$ curves in Fig. 1 and $I_{o}=\exp (B)$ and $W_{d}$ are fitting parameters.

larger than $j_{\mathrm{eo}}\left(T_{e}, n_{e}\right)$. Consequently, (3), which predicts that $V_{\mathrm{pl}} \simeq V_{F}\left(T_{\mathrm{pl}}\right)$ for $R \simeq 1$, is not satisfied in our experiments.

The experimental $I-V$ curves in Fig. 1 were obtained for each emissive probe employed in our experiments for calibration purposes. The probe temperature needs to be raised up to $T_{p}>2200 \mathrm{~K}$ because, for lower values, the curves could not be disclosed by the poor signal-to-noise ratio. The repelled thermionic current for $V_{p}<V_{\mathrm{pl}}$ appears as an effective ion current incrementing with $T_{p}$ that grows for $V_{p}>V_{\mathrm{pl}}$ up to the flat current $I_{\mathrm{co}}\left(T_{p}\right)$. This saturation current characterizes the magnitude of the probe electron current and is independent of further increments in $V_{p}$. This dependence of $I_{\mathrm{co}}\left(T_{p}\right)$ with the wire temperature $T_{p}$ in Fig. 1 has been previously reported by different authors but has not received a fully satisfactory explanation yet [14]. As proposed in [10], Fig. 5 shows the expression for $I_{\mathrm{co}}\left(T_{p}\right)$ that could be approximated by

$$
I_{\mathrm{co}}\left(T_{p}\right)=I_{\mathrm{es}}+I_{o} \exp \left(-\frac{e W_{d}}{k_{B} T_{p}}\right) .
$$

Current $I_{\mathrm{es}}$ represents the electron saturation current $I_{\mathrm{co}}\left(T_{p}\right)$ corresponding to the lower measured thermoelectric electron emission rate, whereas $I_{o}$ and $W_{d}$ are fitting constants. Empirical equation (5) characterizes the response of each emissive probe and measures the maximum collected electron current. 


\section{MODEL}

The emissive probe operates in a plasma in local equilibrium with electron Debye length $\lambda_{D}$ where the ion and electron densities are $n_{i}$ and $n_{e}$, respectively. Both charged species have Maxwellian energy distribution functions with ion $k_{B} T_{i}$ and electron $k_{B} T_{e} \gg k_{B} T_{i}$ temperatures. The emissive Langmuir probe is made of a thin metallic wire with diameter $d \ll \lambda_{D}$ and length $L \gg \lambda_{D}$ heated up to the temperature $T_{p}$.

As (3) our model is restricted to negative bias potentials $\phi=$ $V_{p}-V_{\mathrm{pl}} \leq 0$, therefore, the electrons are repelled from the probe while a small ion current density $j_{\mathrm{ci}}\left(\phi, T_{i}\right)$ is attracted. The fraction of the plasma electrons with kinetic energy $E \geq$ $-e \phi$ overcomes the potential barrier to reach the probe surface. This collected electron current density for $\phi<0$ is given by

$$
j_{\mathrm{ce}}\left(\phi, T_{e}\right)=j_{\mathrm{eo}}\left(T_{e}, n_{e}\right) \exp \left(\frac{e \phi}{k_{B} T_{e}}\right) .
$$

The attracted ion current density $j_{\mathrm{ai}}\left(\phi, T_{i}\right)$ for $\phi<0$ will be neglected in the present model. Its magnitude is small compared with $j_{\mathrm{ce}}\left(\phi, T_{e}\right)$ because of the large ion inertia that brings to $j_{\mathrm{ci}}\left(0, T_{i}\right) \ll j_{\mathrm{ce}}\left(0, T_{e}\right)$. For positive bias $\phi>0$, the attracted electron current density $j_{\mathrm{ae}}\left(\phi, T_{e}\right)$ of plasma electrons depends on their orbital motion and the probe geometry, but its explicit expression is not necessary for the following discussion.

The thermionic electron current density $j_{\mathrm{rd}}\left(T_{p}\right)$ could be considered as a Maxwellian electron group with temperature $2 k_{B} T_{p}$ [1]. The current density of thermionic electrons $j_{\text {thr }}\left(\phi, T_{p}\right)$ rejected by the probe for $\phi<0$ may be approximated by

$$
j_{\mathrm{thr}}\left(\phi, T_{p}\right)=+j_{\mathrm{rd}}\left(T_{p}\right)\left(1-e^{e \phi / 2 k_{B} T_{p}}\right) .
$$

Then, $j_{\text {thr }}\left(\phi, T_{p}\right) \simeq+j_{\text {rd }}\left(T_{p}\right)$ for $\phi \ll 0$ when all electrons are repelled toward the plasma, whereas $j_{\text {thr }}\left(0, T_{p}\right) \simeq 0$ when the probe is biased at the plasma potential $\phi=0$.

This large flow of emitted thermionic electrons from a hot probe, i.e., $1 \ll j_{\text {thr }}\left(\phi, T_{p}\right) / j_{\text {eo }}\left(\phi, T_{e}\right)$ for $\phi \lesssim 0$, interacts with the surrounding plasma and may produce different physical effects. First, as discussed in [6], [7], and [15], the classical plasma sheath around the probe could be modified and might develop a plasma potential minimum acting as a virtual cathode. Hence, a fraction of the initially repelled electrons might return to the emissive probe with a different energy spectrum. Additionally, in partially and fully ionized plasmas, repelled electrons collide with neutrals and/or thermalize with the plasma electrons through Coulomb collisions. Consequently, the initial energy distribution of the repelled thermionic electron group transforms. In the present model, we conjecture that a high energy fraction of initially repelled thermionic electrons might return to the probe that could be collected back as for the plasma electrons.

We are not in position to deduce an explicit expression for this electron density $j_{\text {thc }}$ of repelled electrons that returns to the probe. This would require simultaneously solving both Poisson's and Boltzmann's equations for the ion and electron energy distribution functions. In this phenomenological model, we simply approximate for $\phi \leq 0$ the high-energy thermionic electron group that returns close to the probe surface by a Maxwellian energy distribution with an effective temperature $T_{r}$ as

$$
j_{\text {thc }}\left(\phi, T_{p}, T_{r}\right)=j_{\text {co }}\left(T_{p}\right) e^{e \phi / k_{B} T_{r}} .
$$

As for the plasma electrons, collected only are those with energy enough to overcome the potential barrier. Current density $j_{\mathrm{co}}\left(T_{p}\right)$ approximates the maximum flux of returned electrons that we estimate by using (5) for the empirical fit of $I_{\mathrm{co}}\left(T_{p}\right)$

$$
j_{\mathrm{Co}}\left(T_{p}\right)=j_{\mathrm{es}}+j_{o} \exp \left(-\frac{e W_{d}}{k_{B} T_{p}}\right) .
$$

Constants $j_{e s}, j_{o}$ and $W_{d}$ are obtained from the experimental data fitting and $j_{\mathrm{co}}=I_{\mathrm{co}} / S$. For $\phi \sim 0$ and because $R \gg 1$, we approximate $j_{\text {thc }}\left(0, T_{p}, T_{r}\right) \simeq j_{\text {th }}\left(T_{p}\right)$ while the repelled thermionic electron flux becomes $j_{\text {thr }}\left(\phi, T_{p}\right) \simeq 0$.

For $\phi \lesssim 0$, the emissive probe current density $J_{p}\left(\phi, T_{p}\right)$ is the balance of all these contributions

$$
\begin{aligned}
J_{p}\left(\phi, T_{p}, T_{r}\right)=j_{\mathrm{thc}}\left(\phi, T_{p}, T_{r}\right) & -j_{\mathrm{thr}}\left(\phi, T_{p}\right) \\
& +j_{\mathrm{ce}}\left(\phi, T_{e}\right)-j_{\mathrm{ai}}\left(\phi, T_{i}\right)
\end{aligned}
$$

that recovers for $\phi \sim 0$ the saturation current $I_{\mathrm{co}}\left(T_{p}\right)=S \times$ $j_{\mathrm{co}}\left(T_{p}\right)$ in Fig. 5 as

$$
J_{p}\left(0, T_{p}, T_{r}\right) \simeq-j_{\mathrm{ai}}\left(0, T_{i}\right)+j_{\mathrm{eo}}\left(T_{e}, n_{e}\right)+j_{\mathrm{th}}\left(T_{p}\right)
$$

Neglecting the ion contribution, this last expression considers the cold probe saturation current $j_{\mathrm{eo}}\left(T_{e}, n_{e}\right)$ and a probe temperature-dependent term. Hence, when $T_{p} \simeq 0$, the thermionic emission is negligible and only the plasma electrons contribute to $J_{p}\left(\phi, T_{p}, T_{r}\right)$. On the contrary, when the thermionic emission becomes important, therefore, the knee of the $I-V$ characteristics in Fig. 1 no longer could be identified with $j_{e \circ}\left(T_{e}, n_{e}\right)$ as for cold probes or collecting Langmuir probes.

When the hot probe operates at the floating potential $\phi_{F}=$ $V_{F}-V_{\mathrm{pl}} \leq 0$ setting $J_{p}\left(\phi_{F}, T_{p}, T_{r}\right)=0$ in (7), we have

$$
\begin{aligned}
0=-j_{\mathrm{ai}}\left(\phi_{F}\right. & \left., T_{i}\right)-j_{\mathrm{rd}}\left(T_{p}\right)\left(1-e^{e \phi_{F} / 2 k_{B} T_{p}}\right) \\
& +j_{\mathrm{th}}\left(T_{p}\right) e^{e \phi_{F} / k_{B} T_{r}}+j_{\mathrm{eo}}\left(T_{e}, n_{e}\right) e^{e \phi_{F} / k_{B} T_{e}}
\end{aligned}
$$

where the small contribution of ions $j_{\mathrm{ai}}\left(\phi, T_{i}\right) \ll j_{\mathrm{eo}}\left(T_{e}, n_{e}\right)$ is neglected. Therefore, for a fixed plasma temperature $T_{e}$, the floating potentials $\phi_{F}\left(T_{r}, T_{e}, T_{p}\right)$ are the roots of (8), which are a function of the probe temperature $T_{p}$ and the effective energy $T_{r}$ of the returned electron group collected by the probe.

Classical equation (3) is recovered from (8) under certain assumptions. Separating the probe collected and emitted particle fluxes, we have

$$
\begin{aligned}
j_{\mathrm{ai}}\left(\phi_{F}, T_{i}\right)+j_{\mathrm{rd}}\left(T_{p}\right)\left(1-e^{e \phi_{F} / 2 k_{B} T_{p}}\right) \\
=\left[j_{\mathrm{eo}}\left(T_{e}, n_{e}\right)+j_{\mathrm{th}}\left(T_{p}\right) \times \exp \left(\frac{e \phi_{F}}{k_{B} T_{r}}\left(1-\frac{T_{r}}{T_{e}}\right)\right)\right] . \\
\quad \times e^{e \phi_{F} / k_{B} T_{e}}
\end{aligned}
$$




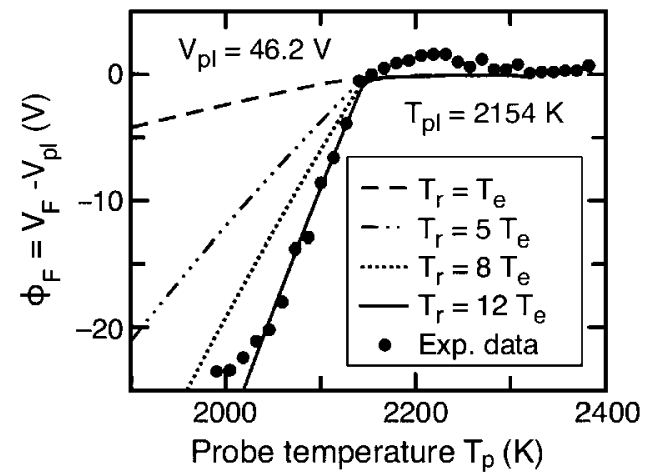

Fig. 6. Comparison between the roots of (8) with the experimental data in the figure for the effective temperatures $T_{r}$ of the additional electron group.

When $T_{e} \geq T_{r}$ and $e\left|\phi_{F}\right| \ll k_{B} T_{e}$, the exponential term could be neglected and because $e\left|\phi_{F}\right| \gg 2 k_{B} T_{p}$, this last expression may be approximated by

$j_{\mathrm{ai}}\left(\phi_{F}, T_{i}\right)+j_{\mathrm{rd}}\left(T_{p}\right)=\left[j_{\mathrm{eo}}\left(T_{e}, n_{e}\right)+j_{\mathrm{th}}\left(T_{p}\right)\right] \times e^{-e\left|\phi_{F}\right| / k_{B} T_{e}}$.

Finally, we obtain

$$
\left|\phi_{F}\right|=\frac{k_{B} T_{e}}{e} \ln \left[\frac{j_{\mathrm{eo}}\left(T_{e}, n_{e}\right)+j_{\mathrm{th}}\left(T_{p}\right)}{j_{\mathrm{ai}}\left(\phi_{F}, T_{i}\right)+j_{\mathrm{rd}}\left(T_{p}\right)}\right]
$$

and the classical expression of (3) is recovered when $j_{\text {th }}\left(T_{p}\right) \simeq 0$. This limit is equivalent to drop in (7) the additional electron group in (6) that is collected back by the probe. Finally, because of the larger values of $j_{\mathrm{th}}\left(T_{p}\right)$ for the transition temperatures $T_{\mathrm{pl}}$, the ratio between the currents in (9) is closer to unity when $R \gg 1$ in (3) [10].

Finally, in order to compare the numerical calculations of the roots of (8) with the experimental data in Fig. 4, two parameters are adjusted, i.e., temperature ratio $T_{r} / T_{e}$ and effective probe emission surface $S_{\text {eff }}$. We represent in Fig. 6 the roots $\phi_{F}\left(T_{r}, T_{e}, T_{p}\right)$ for different ratios $T_{r} / T_{e}$ and $S_{\text {eff }}=S / 8.86$. According to (6), temperature $T_{r}$ measures the energy spread of the second electron group collected by the hot probe and the best fit to $V_{F}\left(T_{p}\right)$ is obtained for $T_{r}=12 T_{e}$. This suggests that the energy distribution of this electron group is non-Maxwellian or has a very broad energy spectrum.

Equation (4) only provides the temperature of the hottest bright probe tip, and an unavoidable temperature gradient exists along the plasma exposed wire. This produces an irregular thermionic emission along the U-shaped wire that could be characterized by means of a reduced effective electron emission surface $S_{\text {eff }}<S$. In Fig. 7, represented is the best fit of the slope for $T_{r}=12 T_{e}$ to the experimental data in Fig. 6 with different effective probe surfaces. The transition temperature $T_{\mathrm{pl}}=2154 \mathrm{~K}$ is obtained for $S_{\mathrm{eff}}=S / 8.86$ (dotted line), which represents $11.3 \%$ of the actual surface $S$ of the probe. The calculations in Fig. 7 show that increments in the effective emission surface of the probe surface reduce the transition temperature.

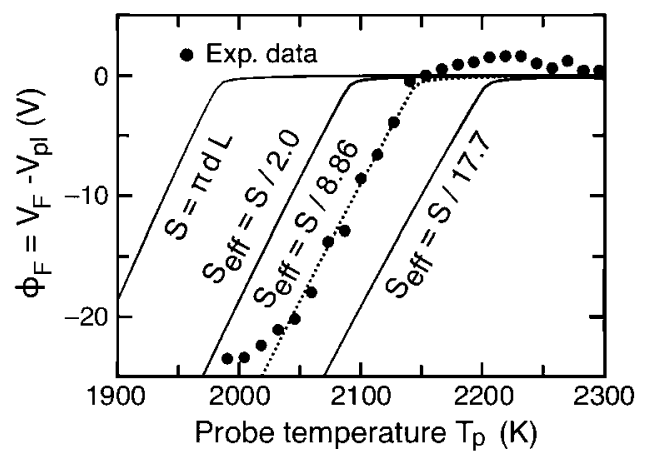

Fig. 7. Effective collecting surface $S_{\text {eff }}$ of the emissive probe for the experimental data in Fig. 6 and $T_{r}=12 T_{e}$. The dashed line corresponds to $S_{\text {eff }}=3.24 \times 10^{-7} \mathrm{~m}^{2}$ equivalent to $11.3 \%$ of the actual surface $S=\pi d L$ of the emissive probe.

\section{CONCLUSION}

We have concluded that $V_{\mathrm{pl}}$ could be determined by using hot emissive Langmuir probes plotting $V_{F}\left(T_{p}\right)$ as a function of $T_{p}$ (or equivalently, probe heating current $I_{H}$ ) as discussed in [8]-[10] and [14]. The abrupt change in the slope of $V_{F}\left(T_{p}\right)$ that appears at temperature $T_{\mathrm{pl}}$, where $V_{F}\left(T_{\mathrm{pl}}\right) \simeq V_{\mathrm{pl}}$, determines the value of the plasma potential. Fig. 3 evidences that the electron-emitted current modifies the probe floating potential for temperatures $T_{p}>2000 \mathrm{~K}$, corresponding to $R>10$ in Fig. 4. Hence, the strong emission regime could be defined from this temperature up to the knee in Fig. 3 at $T_{\mathrm{pl}}$ with $R \sim 10^{2}-10^{3}$ range where electron emission plays a relevant role. Consequently, for $T_{p}>T_{\mathrm{pl}}$, the probe operates beyond the strong emission regime of $R \gg 1$ and outside the validity of (9) of our model.

Equation (3) is not valid in this strong emission regime of operation, and in order to explain this discrepancy, we have derived a phenomenological model only valid for negatively biased probes where $\phi \leq 0$. Our model considers that two electron populations are collected by the probe. The plasma electrons and those coming from the additional electron group are also considered as Maxwellian with temperature $T_{r}$. This simple approximation does not preclude the possibility of more involved energy distribution functions for these electrons. The physical origin of this second electron population might be emerging of virtual cathodes or a potential well in the plasma sheath close to the emissive probe. Such potential structures would return a fraction of the large emitted thermionic electron current for $\phi \leq 0$ with a different energy spectrum. These complex plasma potential profiles around electron-emitting probes were considered in [6], [7], and [15].

The emissive probe provides the energy source required for such electron energization, as well as for such plasma sheath modifications. In our case, the probe transfers to the surroundings about $1.5-4.4 \mathrm{~W}$ of electric power. Despite the radiation and thermal losses, an important fraction of this energy is transported by the large thermionic electron current toward the plasma.

The presence of two electron groups nearby the emissive probe would be confirmed by the good agreement of the experimental data in Fig. 6 with the predicted floating potential, calculated as the roots of (8). This additional electron 
population would explain why the floating potential of the emissive probe at the knee in Fig. 4 provides the local plasma potential, even for $R \gg 1$ when (3) is not valid. The strong thermionic electron emission introduces correction factors leading to (9) in certain limits. These results also question the possibility of determining the electron temperature using (3) or (9) because our model suggests that the slope in Fig. 6 is related with $T_{r}$ instead of $T_{e}$ through a more involved expression related with the roots of $(8)$.

The effective electron emission surface determines the transition temperature $T_{\mathrm{pl}}$ of the probe corresponding to the knee of $V_{F}\left(T_{p}\right)$. The dependence of $T_{\mathrm{pl}}$ with the effective probe surface $S_{\text {eff }}<S$ in Fig. 7 evidences that the thermionic current is not uniform over the emissive probe surface. Additionally, the U-shaped wire is small; only a fraction of its surface directly points toward the plasma, and this would increase the reduction of the effective thermionic current also equivalent to a lower emitting surface. This would explain that probes made of wires with varied diameters provide the same value of $V_{\mathrm{pl}}$ for different heating currents $I_{H}$ [14].

Finally, the recommended values [1] for polycrystalline tungsten are $A=40-100 \mathrm{~A} \mathrm{~cm}^{-2} \mathrm{~K}^{-2}$ in (1), whereas the theoretical value of $A=120 \mathrm{~A} \mathrm{~cm}^{-2} \mathrm{~K}^{-2}$ is about two times higher. This discrepancy depends on factors out of our control in the experiments, including the state of metal surface, impurities, etc. Therefore, we made use of the theoretical value in our calculations because the changes in (1) introduced by factor two in $A$ do not lead to relevant modifications in our conclusions extracted from Figs. 4, 6, and 7.

\section{REFERENCES}

[1] Y. P. Raizer, Gas Discharge Physics. Berlin, Germany: Springer-Verlag, 1991, pp. 103-127.

[2] N. Hershkowitz, "How Langmuir probes work," in Plasma Diagnostics Discharge Parameters and Chemistry, O. Auciello and D. L. Flamm, Eds. London, U.K.: Academic, 1989, pp. 113-183.

[3] J. P. Sheehan and N. Hershkowitz, "Emissive probes," Plasma Sources Sci. Technol., vol. 20, no. 6, pp. 063001-1—063001-21, Dec. 2011.
[4] J. P. Sheehan, Y. Raitses, N. Hershkowitz, I. Kaganovich, and N. J. Fisch, "A comparison of emissive probe techniques for electric potential measurements in a complex plasma," Phys. Plasmas, vol. 18, no. 7, pp. 073501-1-073501-9, Jul. 2011.

[5] J. R. Smith, N. Hershkowitz, and P. Coakley, "Inflection-point method of interpreting emissive probe characteristics," Rev. Sci. Instrum., vol. 50, no. 2, pp. 210-218, Feb. 1979.

[6] S. Takamura, N. Ohno, M. Y. Ye, and T. Kubawara, "Space-charge limited current from plasma facing material surface," Contrib. Plasma Phys., vol. 44, no. 13, pp. 126-137, Apr. 2004.

[7] M. Y. Ye and S. Takamura, "Effect of space-charge limited emission on measurements of plasma potential using emissive probes," Phys. Plasmas, vol. 7 , no. 8, pp. 3457-3463, Aug. 2000.

[8] R. F. Kemp and J. M. Sellen, "Plasma potential measurements by electron emissive probes," Rev. Sci. Instrum., vol. 37, no. 4, pp. 455-464, Apr. 1966.

[9] P. Balan, R. Schrittwieser, C. Ionita, J. A. Cabral, H. F. C. Figueiredo, H. Fernandes, C. Varandas, J. Adamek, M. Hron, J. Stockel, E. Martines, M. Tichy, and G. Van Oost, "Emissive probe measurements of plasma potential fluctuations in the edge plasma regions of tokamaks," Rev. Sci. Instrum., vol. 74, no. 3, pp. 1583-1587, Mar. 2003.

[10] S. P. Tierno, E. Roibás, J. L. Doménech-Garret, J. M. Donoso, and L. Conde, "Measurements of the plasma properties using the floating potential of emissive Langmuir probes," Contrib. Plasma Phys., vol. 53, no. 1, pp. 16-21, Jan. 2013.

[11] I. Langmuir, "The characteristics of tungsten filaments as functions of temperature," Phys. Rev., vol. 7, no. 3, pp. 302-330, Mar. 1916.

[12] E. Criado, E. Roibás, S. P. Tierno, P. Rodríguez De Francisco, J. L. Domenech-Garret, J. M. Donoso, and L. Conde, "Ion beam neutralization and properties of plasmas from low power ring cusp ion thrusters," Phys. Plasmas, vol. 19, no. 2, pp. 023505-1-023505-8, Feb. 2012.

[13] O. Troll, L. Conde, E. Criado, J. M. Donoso, and G. Herdrich, "Measurements of plasma properties using fast sweep Langmuir probes in unmagnetized weakly ionized plasmas," Contrib. Plasma Phys., vol. 50, no. 9, pp. 819-823, Sep. 2010

[14] A. Marek, I. Picková, P. Kudrna, M. Tichánd, P. R. Apetrei, S. B. Olenici, S. Gstrein, R. Schrittwieser, and C. Ionita, "Experimental investigation of the change of the electron saturation current of a DC heated emissive probe," Czech. J. Phys., vol. 56, no. 2, pp. B932-B937, Oct. 2006.

[15] T. Gyergyek, B. Jurèiè-Zlobec, M. Ėerèek, and J. Kovaèiè, "Sheath structure in front of an electron emitting electrode immersed in a two electron temperature plasma: A fluid model and numerical solutions of the Poisson equation," Plasma Sources Sci. Technol., vol. 18, no. 3, pp. 035001-1035001-18, Aug. 2009. 